\title{
Prof. Dr. med. Thomas G. Wendt Direktor der Klinik für Strahlentherapie und Radioonkologie des Universitätsklinikums Jena zum 65. Geburtstag
}

\author{
Tilo Wiezorek · Gerhard G. Grabenbauer · Rolf Sauer
}

Online publiziert: 2. Oktober 2014

(C) Springer-Verlag Berlin Heidelberg 2014

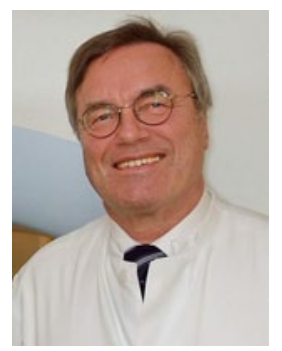

Professor Thomas Wendt (Michael Szabo/Uniklinikum Jena mit freundlicher Genehmigung)

In der Landeshauptstadt München, am 20. August im Jahre 1949 geboren, verbrachte Thomas Wendt als echtes „Münchner Kindl“" seine Schulzeit am Ruprecht-Gymnasium in München-Neuhausen und absolvierte das Studium an der weltbekannten, heimischen Ludwig-MaximiliansUniversität. Die Facharztweiterbildung, seinerzeit noch zum ,großen“" Radiologen, führte ihn nach kurzen Umwegen relativ zügig wieder zurück an seine Alma Mater, wo er unter dem (stets wegen des grünen Korrekturstifts)

Dr.-Ing. T. Wiezorek ( $\bowtie)$

Klinik für Strahlentherapie und Radioonkologie,

Universitätsklinikum Jena,

Bachstr. 18,

07740 Jena, Deutschland

E-Mail: Tilo.Wiezorek@med.uni-jena.de

G. G. Grabenbauer

Klinik für Strahlentherapie und Radioonkologie,

Klinikum Coburg,

Coburg, Deutschland

R. Sauer

Universitätsklinikum Erlangen,

Erlangen, Deutschland

geschätzten Prof. Josef Lissner eine umfassende wissenschaftliche wie klinische Qualifikation erlangte, die heute sicher Ihresgleichen sucht. Den Weg säumten seinerzeit die nicht unbekannten Kollegen Moser, Kirsch, Eiermann, Vogl und Hahn. Später, als der wissenschaftliche Fokus bereits längst der Radioonkologie galt, kamen erfolgreiche wissenschaftliche und klinische Kollaborationen mit den Kollegen Willich, Rohloff, Wustrow und Trott hinzu, sodass die Habilitation im Jahre 1990, naturgemäß zur Radiochemotherapie des inoperablen Kopf-Hals-Tumors, nur allzu logisch folgte. Mit höchstem Respekt ist zu konstatieren, dass dieses Behandlungsprinzip zwar nicht nur durch Thomas Wendt „erfunden“ wurde, aber er schaffte es als erster, konsequent klinische Studien durchzuführen und sie im seinerzeit (für Radioonkologen) noch unerreichbaren Journal of Clinical Oncology mehrfach zu publizieren.

Als Professor Wendt im Herbst 1994 die Leitung der Klinik für Strahlentherapie und Radioonkologie des Universitätsklinikums Jena übernahm, fand er eine nicht mehr ganz moderne technische Basis vor. Sie bestand zum einen aus einem russischen Linearbeschleuniger LUE, dessen Gantry lediglich um max. $+/-90^{\circ}$ schwenkbar war und bei dem die Umstellung von Elektronen auf Photonen ca. $30 \mathrm{~min}$ inklusive Messung erforderte. Zum anderen bestand sie aus zwei Telekobalt-Anlagen und einem (eben frisch installierten) neuen Siemens-Beschleuniger KD2. Für die Brachytherapie stand ein Gammamed 12i zur Verfügung, welches vornehmlich für intraluminale Bestrahlungen verwendet wurde. Abgesehen von diesen etwas eingeschränkten Möglichkeiten fand er jedoch ein erfahrenes Klinikteam vor, das neben den Poliklinikpatienten auch die Patienten von drei Stationen mit insgesamt 64 Betten versorgte. Zu dieser Zeit wurden in Jena bereits ca. $20 \%$ der zu behandelnden Fälle mit ,21/2-D-Berechnung“", basierend auf 3-4 händisch digitalisierten CT-Einzelscans, geplant. Des Weiteren wurden 
damals routinemäßig Patienten vor Knochenmarktransplantation mit einer speziellen Großfeldtechnik am Telekobalt behandelt.

Zum Professor für Strahlentherapie an der FriedrichSchiller-Universität wurde Professor Wendt im Mai 1996 ernannt. Stets optimierte er seine Vorlesungsskripte, angepasst an den neuesten technischen Stand, um angehende Mediziner für den spannenden Beruf des Strahlentherapeuten zu begeistern.

Neben seiner Arbeit als Hochschullehrer und Wissenschaftler nahm für Prof. Wendt das Vorantreiben der technologischen Modernisierung der Klinik zur Verbesserung der Therapie seiner Patienten eine herausgehobene Stellung ein. Dem kam sein außerordentliches technisch-mathematisches Verständnis sehr entgegen. Da nicht alle Einzelheiten genannt werden sollen, hier nur einige Meilensteine:

1996: Einführung der 3-D-Planung mit Helax TMS

1997: TBI mit Translationsliege am Telekobalt

1998: Beginn stereotaktisch geführter Radiochirurgie mit Rundlochkollimatoren

1998: Einbindung von MRT-Datensätzen in die Bestrahlungsplanung

1999: Beginn stereotaktisch geführter RT mit MicroMLC m3 (3-mm-Leafs)

2001: Vorwärts geplante IMRT mit step \& shoot (1-cm-Leafs)

2001: Invers geplante IMRT mit Kompensatoren

2003: Inverse geplante IMRT mit step \& shoot (1-cm-Leafs)

2004: Einbindung von PET-Datensätzen in die Bestrahlungsplanung

2006: Einführung atemgetriggerter Bestrahlung

2007: Invers geplante IMRT mit step \& shoot mit MicroMLC m3

2008: Interstitielle Brachytherapie der Mamma, Gammamed Plus

2009: Hochauflösende MLCs (5-mm-Leafs) an beiden Linac (Tandembetrieb)

2011: Einführung IGRT für Patienten mit hypofraktionierter Bestrahlung

2012: Beginn der hypofraktionierten „Bodystereotaxie“ bei Lungenmetastasen
2013: Beginn der hypofraktionierten „Bodystereotaxie“ bei Lebermetastasen

2013: Adaptive Strahlentherapie bei verschiedenen Indikationen am Tomotherapiesystem

2014: Ganzkörperbestrahlungen am Tomotherapiesystem

Das besondere Interesse stets neue, schonendere und präzisere Verfahren für seine Patienten anbieten zu können und dies gleichzeitig wissenschaftlich zu begleiten, äußerte sich in zwei Formen. Eine davon war und ist die Betreuung junger Promovenden. Für 27 junge Ärzte und Medizinphysiker stand er in Jena als Doktorvater zur Verfügung. Eine andere Form war die Wahrnehmung der Präsidentschaft und aller damit verbundenen Gestaltungsmöglichkeiten bei der Ausrichtung der DEGRO-Jahrestagung 2004 in Erfurt und 2 Jahre später die wissenschaftliche Organisation einer kleinen, aber hochspezialisierten Tagung „Moderne Technologien in der Strahlentherapie" auf den Dornburger Schlössern, mit großer Resonanz aus der Strahlentherapie-Community.

Professor Wendts sonstige wissenschaftliche Aktivitäten sind vielfältig und auf das ganze Spektrum der Strahlentherapie ausgedehnt. Sie manifestieren sich in diversen Publikationen: Originalarbeiten, Übersichtsarbeiten und Buchbeiträgen. Sein Engagement in der Deutschen Krebsgesellschaft gipfelte darin, dass er von 1999 bis 2003 den Vorsitz der Arbeitsgemeinschaft Radiologische Onkologie (ARO) innehatte. Professor Wendt engagierte sich stark für das Jenaer Tumorzentrum und leitete es von 2005 bis 2008. Zur Optimierung der Versorgung von Tumorpatienten auch aus den peripheren Häusern initiierte er nach und nach sehr erfolgreich Tumorkonferenzen zu verschiedenen Tumorentitäten.

Thomas Wendt, einer der letzten „Dinosaurier der deutschen Radioonkologie“, der an all seinen Wirkungsstätten nachhaltige Fußabdrücke hinterlassen hat, beging im August dieses Jahres seinen 65. Geburtstag. Wir wünschen ihm, dem verehrten Lehrer und Freund, mit Rainer Maria Rilke „Worte des Segens und den unendlichen Namen“.

Tilo Wiezorek, Gerhard G. Grabenbauer und Rolf Sauer, Jena/Coburg/Erlangen 
Hier steht eine Anzeige.

黛 Springer 